\title{
CD146 controls the quality of clinical grade mesenchymal stem cells from human dental pulp
}

\author{
Lan $\mathrm{Ma}^{\dagger}$, Zhiqing Huang ${ }^{\dagger}$, Di Wu, Xiaoxing Kou, Xueli Mao and Songtao Shi
}

\begin{abstract}
Background: Human mesenchymal stem cells from dental pulp (hMSC-DP), including dental pulp stem cells from permanent teeth and exfoliated deciduous teeth, possess unique MSC characteristics such as expression of specific surface molecules and a high proliferation rate. Since hMSC-DP have been applied in numerous clinical studies, it is necessary to establish criteria to evaluate their potency for cell-based therapies.
\end{abstract}

Methods: We compared stem cell properties of hMSC-DP at passages 5, 10 and 20 under serum (SE) and serum-free (SF) culture conditions. Cell morphology, proliferation capacity, chromosomal stability, surface phenotypic profiles, differentiation and immunoregulation ability were evaluated. In addition, we assessed surface molecule that regulates hMSC-DP proliferation and immunomodulation.

Results: hMSC-DP exhibited a decrease in proliferation rate and differentiation potential, as well as a reduced expression of CD146 when cultured under continuous passage conditions. SF culture conditions failed to alter surface marker expression, chromosome stability or proliferation rate when compared to SE culture. SF-cultured hMSCDP were able to differentiate into osteogenic, adipogenic and neural cells, and displayed the capacity to regulate immune responses. Notably, the expression level of CD146 showed a positive correlation with proliferation, differentiation, and immunomodulation, suggesting that CD146 can serve as a surface molecule to evaluate the potency of hMSC-DP. Mechanistically, we found that CD146 regulates proliferation and immunomodulation of hMSC-DP through the ERK/p-ERK pathway.

Conclusion: This study indicates that SF-cultured hMSC-DP are appropriate for producing clinical-grade cells. CD146 is a functional surface molecule to assess the potency of hMSC-DP.

Keywords: Human dental pulp stem cells, Quality, Clinical grade, CD146, ERK pathway

\section{Introduction}

Mesenchymal stem cells (MSCs) have been used in clinics to treat a variety of human diseases [1-4]. MSCs can be isolated from multiple tissues, such as bone marrow,

*Correspondence: shisongtao@mail.sysu.edu.cn

${ }^{\dagger}$ Lan Ma and Zhiqing Huang have contributed equally to this work South China Center of Craniofacial Stem Cell Research and Guangdong Province Key Laboratory of Stomatology, Guanghua School and Hospital of Stomatology, Sun Yat-Sen University, Guangzhou 510055, Guangdong, People's Republic of China umbilical cord tissue, adipose tissue and dental pulp [5-7]. The minimal criteria for MSC identification were established by the International Society for Cellular Therapy (ISCT) in 2006 [8]. However, the standards for the quality assessment of MSCs from specific tissue resources have not yet been reported.

Human mesenchymal stem cells from dental pulp (hMSC-DP) have been isolated and extensively studied $[9,10]$. Their superior proliferation, multi-differentiation, and immunomodulatory capacities have been reported [11-13]. Recently, hMSC-DP have been applied original author(s) and the source, provide a link to the Creative Commons licence, and indicate if changes were made. The images or other third party material in this article are included in the article's Creative Commons licence, unless indicated otherwise in a credit line to the material. If material is not included in the article's Creative Commons licence and your intended use is not permitted by statutory regulation or exceeds the permitted use, you will need to obtain permission directly from the copyright holder. To view a copy of this licence, visit http://creativecommons.org/licenses/by/4.0/. The Creative Commons Public Domain Dedication waiver (http://creativeco mmons.org/publicdomain/zero/1.0/) applies to the data made available in this article, unless otherwise stated in a credit line to the data. 
to regenerate orofacial tissues and treat systemic diseases in pre-clinical and clinical settings [11, 14-18]. It is well-known that the quality and potency of MSCs are critical to ensure their therapeutic effects [19]. However, the minimum criteria for MSCs formulated by ISCT may not provide enough guidance to evaluate the quality and potency of hMSC-DP [8].

Previous studies indicate that fetal bovine serum (FBS)cultured MSCs may lead to some ethical and immune response issues [20-22]. Obvious quality variation across FBS batches also limits the large-scale manufacture of MSCs. Therefore, it is necessary to use serum-free (SF) culture systems for the future use of MSCs in clinics. FBS substitutes, including human platelet lysate and chemically defined SF media, appear to provide sufficient nutritional support for the culture expansion of hMSC-DP and maintain their regenerative potency [23-25]. However, detailed cultural effects of SF medium on hMSC-DP are not fully understood.

In this study, we established a SF culture system suitable for the evaluation of culture-expanded hMSC-DP. Additionally, we identified that CD146 is a potential functional surface molecule to show the potency of hMSC-DP and established minimal criteria to assess the potency of hMSC-DP.

\section{Materials and methods \\ Mice}

Male C57BL/6J mice were purchased from Sun Yat-sen University, Guangzhou, China. All animal experiments were performed under an institutionally approved protocol for the use of animal research (Sun Yat-sen University, SYSU-IACUC-2020-000394).

\section{Isolation and culture of hMSC-DP}

Human exfoliated deciduous incisors and third molars were obtained as discarded biological samples from Guanghua School and Hospital of Stomatology, Sun Yat-sen University. The informed consent forms were acquired from each guardian on behalf of the child donors or from each adult donor, respectively. All teeth were intact without any inflammation. The protocol (KQEC-2020-055-01) was approved by the Medical Ethics Committee of Hospital of Stomatology, Sun Yat-sen University. DPSCs and SHED were isolated and cultured until passage 2 (P2) as reported previously [9, 10]. P2 hMSC-DP were cultured with serum (SE) or serumfree (SF) conditions until P20. SE-hMSC-DP were cultured as previously reported $[9,10]$. SF-hMSC-DP were cultured in a high-glucose Dulbecco's Modified Eagle Medium (DMEM, Invitrogen) supplemented with 5\% human platelet lysate (Compass Biomedical), $100 \mathrm{U} /$ $\mathrm{ml}$ penicillin, $100 \mathrm{mg} / \mathrm{ml}$ streptomycin (Gibco), and $1 \%$
GlutaMAX $^{\mathrm{TM}}$ Supplement (Gibco). The plastic-adherent confluent cells were passaged with $0.25 \%$ trypsin containing $1 \mathrm{mM}$ EDTA (Gibco) and continuously maintained in the SE or SF medium. Approximately $1 \times 10^{6} \mathrm{hMSC}$ DP were selected for inoculation on a $10 \mathrm{~cm}$ culture dish (Corning) at each passage, while the remaining cells were stored in serum-free cryopreservation medium (CELLBANKER $^{\mathrm{TM}}$ 2) at $-80{ }^{\circ} \mathrm{C}$. hMSC-DP at P5, P10 and P20 were used for subsequent tests.

\section{Antibodies and reagents}

Anti-CD34, CD45, CD73, CD90, CD105, CD146 and 7-AAD antibodies were purchased from BD Biosciences. Anti-CD3, CD8 and Annexin V antibodies were purchased from BioLegend. Anti-Runx2, ERK and p-ERK antibodies were purchased from Cell Signaling Technology. Anti-CD146, ALP, $\beta$ III-tubulin and NeuN antibodies were purchased from Abcam. Anti-LPL and $\beta$-actin antibodies were purchased from Invitrogen. Anti-PPAR $\gamma$ antibody was purchased from Santa Cruz. WGA-488 was purchased from Biosharp. Hoechst 33,342 was purchased from Sigma. The kFluor488-EDU cell proliferation assay kit was purchased from KeyGEN Biotech. Lipofectamine $^{\mathrm{TM}}$ RNAiMAX transfection reagent was purchased from Invitrogen. CD146 siRNA was purchased from RIBO Biotech. PD98059 was purchased from Beyotime. Cellular Senescence Assay Kit was purchased from Merck Millipore. Dextran Sulfate Sodium (DSS) was purchased from MP Biochemicals.

\section{Flow cytometry analysis}

For cell surface marker analysis, hMSC-DP suspension from P5, P10 and P20 were prepared at a density of $10^{6}$ cells per $100 \mu \mathrm{l}$. Thereafter, $1 \mu \mathrm{l}$ antibody was added to $100 \mu \mathrm{l}$ cell suspension and incubated for $30 \mathrm{~min}$ at $4{ }^{\circ} \mathrm{C}$ in the dark, followed by analysis using flow cytometry (ACEA NovoCyte $^{\mathrm{TM}}$ ). The apoptotic $\mathrm{T}$ cells were stained with $\mathrm{CD} 3$ and 7AAD antibodies at $4{ }^{\circ} \mathrm{C}$ in the dark for $30 \mathrm{~min}$ and washed twice with PBS, followed by incubation with Annexin V antibody.

\section{In vitro immunomodulatory capacity of hMSC-DP}

hMSC-DP at P5, P10 and P20 $\left(1 \times 10^{5} /\right.$ per well $)$ were seeded on a 12-well culture plate (Corning) and incubated for $24 \mathrm{~h}$. T cells, pre-stimulated by anti-CD3 and CD8 antibodies, were directly loaded onto hMSC-DP and cocultured for 2 days. The number of apoptotic $\mathrm{T}$ cells was detected by flow cytometry.

\section{Karyotype analysis}

Karyotype analysis of hMSC-DP, including chromosome numbers and G-banded karyotypes, was performed by 
using a kit from HaoGe Biotechnology Corporation Limited (Shanghai, China).

\section{EdU incorporation assay}

hMSC-DP $\left(2 \times 10^{4}\right.$ cells per well $)$ were seeded in 24-well plates (Corning) and incubated at $37{ }^{\circ} \mathrm{C}$ for $24 \mathrm{~h}$. Cells were treated with 5-ethynyl-20-deoxyuridine (EdU) at a working concentration of $50 \mu \mathrm{M}$ in a $200 \mu$ culture medium for $2 \mathrm{~h}$. Then cells were detected using an EdU cell proliferation assay kit (KeyGEN BioTECH) according to the manufacturer's instructions.

\section{Population doublings}

hMSC-DP at P4 were seeded at $2 \times 10^{5}$ cells in $60 \mathrm{~mm}$ dishes (Corning) in SE or SF culture medium. Cells were harvested and seeded at the same number when they reached around $70-80 \%$ confluence. Population doublings (PD) were calculated by the following formula: $\mathrm{PD}=\log 2$ (number of harvested cells/number of seeded cells). PD numbers were determined by the cumulative addition of total numbers generated from P5 to the end of cell division. The PD assay was repeated with 3 independent isolated cells for each group.

\section{Colony-forming unit (CFU) assay}

A total number of 1000 hMSC-DP from each group were cultured and plated in $6 \mathrm{~cm}$ dishes (Corning). After 10 days, cells were fixed with $4 \%$ paraformaldehyde and stained with $1 \%$ crystal violet for $5 \mathrm{~min}$. Colonies containing 50 or more cells were selected. The experiment was repeated three times.

\section{Cell morphology analysis by high-content imaging} 3000 hMSC-DP of each group were seeded in 96-cell well plates (Corning) and incubated for $24 \mathrm{~h}$. Adherent cells were washed with PBS and then fixed in $4 \%$ formaldehyde for $10 \mathrm{~min}$ at room temperature. Cells were stained with Phalloidin, WGA, and Hoechst 33,342. Plates were scanned in the Operetta CLS and cells of each group were analyzed for cell size and shape using the Harmony software (PerkinElmer).

\section{Cellular senescence assay}

A total number of $5 \times 10^{4}$ hMSC-DP from each group were seeded in 24-well plates (Corning) and incubated for $24 \mathrm{~h}$. Then a cellular senescence assay was conducted following the protocol provided with the Cellular Senescence Assay Kit (Merck Millipore).

\section{Induction of osteogenic, adipogenic and neurogenic differentiation}

Approximately $3 \times 10^{5} \mathrm{hMSC}$-DP of each group were cultured in $6 \mathrm{~cm}$ plates until they reached full confluence.
The osteogenic and adipogenic differentiation induction and assessment were conducted as reported previously $[26,27]$. For neurogenic differentiation, a total of $4 \times 10^{4}$ cells was seeded on a 24-well culture plate (Corning) and incubated for $24 \mathrm{~h}$. Then cells were changed to neural differentiation medium and cultured for 10 days. The neural differentiation assessment was conducted as reported previously [10].

\section{siRNA transfection and chemical treatment}

For siRNA transfection, hMSC-DP $\left(5 \times 10^{5} /\right.$ well $)$ were seeded in a 6-well culture plate in a low-serum medium (Opti-MEM, Gibco) and treated with CD146 siRNA (RIBO, China) or control vehicle siRNA with lipofectamine reagent (Invitrogen) for $24 \mathrm{~h}$, according to the manufacturer's instructions. For chemical reagent treatments, hMSC-DP were treated with $10 \mu \mathrm{M}$ PD98059. Treated cells were harvested or used for further experiments.

\section{Allogenic hMSC-DP transplantation in acute colitis mice} Acute colitis was induced by administering 3\% DSS (MP Biochemicals) dissolved in drinking water for 8 days. A total number of $1 \times 10^{6} \mathrm{hMSC}$-DP from each group were infused into colitis mice intravenously at day 3 after feeding with DSS water. All mice were euthanized and analyzed on day 8 . Induced colitis mice were evaluated as previously described [28].

\section{Statistical analysis}

GraphPad Prism 8 was used to perform statistical analysis. Comparisons between two groups were analyzed using independent two-tailed Student's $t$ tests, while comparisons between more than two groups were analyzed using one-way ANOVA. $P$ values $<0.05$ were considered statistically significant.

\section{Results \\ Morphological characteristics of hMSC-DP}

We isolated DPSCs from the third molars of three adult donors and SHED from deciduous incisors of three 6-8 years old donors. All donors were healthy volunteers and the teeth were devoid of caries and inflammation. hMSC-DP, including DPSCs and SHED, were isolated from dental pulp according to the previous reports [9, 10]. hMSC-DP at P2 were separated to be propagated under traditional FBS (SE) or human platelet lysate serum-free (SF) culture conditions to generate DPSC-SE, DPSC-SF, SHED-SE and SHED-SF (Fig. 1a).

Cell morphology is regarded as an MSC potency indicator [29]. hMSC-DP from all donors under SE and SF culture conditions adhered to culture dishes and showed elongated and spindle-shaped morphology at P5 and P10. 
(See figure on next page.)

Fig. 1 Morphological characteristics of hMSC-DP with accumulated passages. a Schema of isolation and culture of hMSC-DP under SE or SF culture conditions. b The morphology of P5, P10 and P20 hMSC-DP under SE or SF culture conditions. Scale bar =50 um. c Cell morphology analysis by high-content imaging. hMSC-DP were co-stained with Phalloidin (orange), Hoechst (blue) and WGA (green). Scale bar $=100 \mu \mathrm{m}$. $n=3$ for each group. $\mathbf{d}$ Cellular senescence assay. The percentage of SA- $\beta$-gal positive cells was calculated and compared across groups at P5, P10 and P20. The black arrow indicates positive staining of SA- $\beta$-gal. Scale bar $=100 \mu \mathrm{m} . n=3 \sim 5$ for each group. SE, serum; SF, serum-free. Data shown as mean \pm SEM. ${ }^{*} p<0.05,{ }^{* *} p<0.01,{ }^{* * *} p<0.001$. ns not significant

With continuous passage, hMSC-DP became enlarged, cuboidal, and flattened at P20 (Fig. 1b). To further analyze cellular morphological characteristics, we used a high-content imaging approach to show Hoechst-positive nuclei, Phalloidin-positive cytoskeleton and wheat germ agglutinin (WGA)-positive cell membrane [30]. More than 500 cells of each group were analyzed. The nuclear size of hMSC-DP at P20 was significantly larger than P5 in the DPSC-SE $(P<0.01)$, DPSC-SF $(P<0.05)$, SHED-SE $(P<0.05)$ and SHED-SF $(P<0.01)$ groups. The cell size of hMSC-DP at P20 also showed a tendency to become larger when compared to hMSC-DP at P10 (Fig. 1c). The nuclear size showed no significant difference between SE and SF culture conditions (Additional file 1: Fig. S1a), but the cell size of SHED tended to be smaller in SF culture compared to SE culture $(P<0.05$ at $\mathrm{P} 10, P<0.01$ at $\mathrm{P} 20)$ (Additional file 1: Fig. S1b). Since cell senescence may be associated with alteration of cellular and nuclear size [31], we assessed $\beta$-galactosidase ( $\beta$-gal)-positive senescent hMSC-DP at P5, P10 and P20. We showed that the percentage of $\beta$-gal positive cells increased from P5 to P20 in each group, which was statistically significant when compared between P5 hMSC-DP and P20 hMSCDP $(P<0.001$ in each group) (Fig. $1 \mathrm{~d})$. However, there was no statistically significant difference between SE and SF culture conditions (Additional file 1: Fig. S1c). These data indicate that continuous passage affects hMSC-DP morphological characteristics.

\section{Proliferation capacity and chromosomal stability of hMSC-DP}

Proliferation capacity is an important parameter for evaluating the potency of MSCs. hMSC-DP were seeded at a density of 1,000 cells/plate and cultured for 10 days. The number of colonies was significantly decreased from P5 to P20 (Fig. 2a). Interestingly, the SHED-SE group showed a higher number of colony formation compared to the SHED-SF group at P20 (Additional file 1: Fig. S2a). To examine the proliferation potential of hMSC-DP, population doubling scores were calculated from three donorderived batches of DPSCs and three donor-derived batches of SHED. These cells showed similar proliferation capacities (Fig. 2b) and had no significant differences under either SE or SF culture conditions (Additional file 1: Fig. S2b). Also, 5-Ethynyl-2'-deoxyuridine (EdU) staining showed the proliferation rate of hMSC-DP decreased with the continuous passage, and significantly reduced at P20 compared to P5 (Fig. 2c) $(P<0.001$ at each group). SE and SF culture showed no differences in terms of EdU incorporation (Additional file 1: Fig. S2c).

It is critical to use clinical-grade human MSCs with normal genetic karyotypes and chromosomal stability [32]. We assessed the karyotypes of DPSCs and SHED at P5, P10 and P20 under SE and SF culture conditions. There was no detectable chromosomal aberration in any of the tested groups (Fig. 2d), consistent with the previous reports that human MSCs maintain relatively stable genomes during culture expansion [33, 34].

\section{Surface phenotypic profiles and in vitro immunoregulation ability of hMSC-DP}

We next analyzed a series of surface markers including those described by the ISCT [8]. hMSC-DP at P5, P10 and P20 under both SE and SF culture conditions expressed MSC surface markers CD73, CD90 and CD105 while they did not express hematopoietic markers CD34 and CD45 (Fig. 3a). Notably, the expression level of CD146 exhibited heterogeneity across groups (Fig. 3a, Additional file 1: Fig. S3a). We observed a gradually decreased expression of CD146 from P5 to P10 as well as from P10 to P20. There was a significant reduction of CD146 expression at P20 when compared to P5 (DPSC-SE, DPSC-SF, SHED-SE and SHED-SF: $P<0.001$ ) (Fig. 3b). Neither SE nor SF culture conditions showed a regulatory effect on the expression of CD146 (Additional file 1: Fig. S3b). Previous studies showed that CD146 is not only a melanoma cell adhesion molecule but also a cellular surface receptor of various ligands, participating in numerous physiological and pathological processes [35]. Importantly, CD146 has been identified as an MSC surface marker [36].

To assess the in vitro immunomodulatory capacity of hMSC-DP, we co-cultured hMSC-DP with activated T cells for 2 days. Flow cytometry analysis showed that hMSC-DP at P5 and P10 were capable of inducing a significant number of $\mathrm{T}$ cell apoptosis compared to the control group, but hMSC-DP at P20 lost their capacity to induce $\mathrm{T}$ cell apoptosis (Fig. 3c). hMSC-DP under SF culture condition showed a tendency to possess 


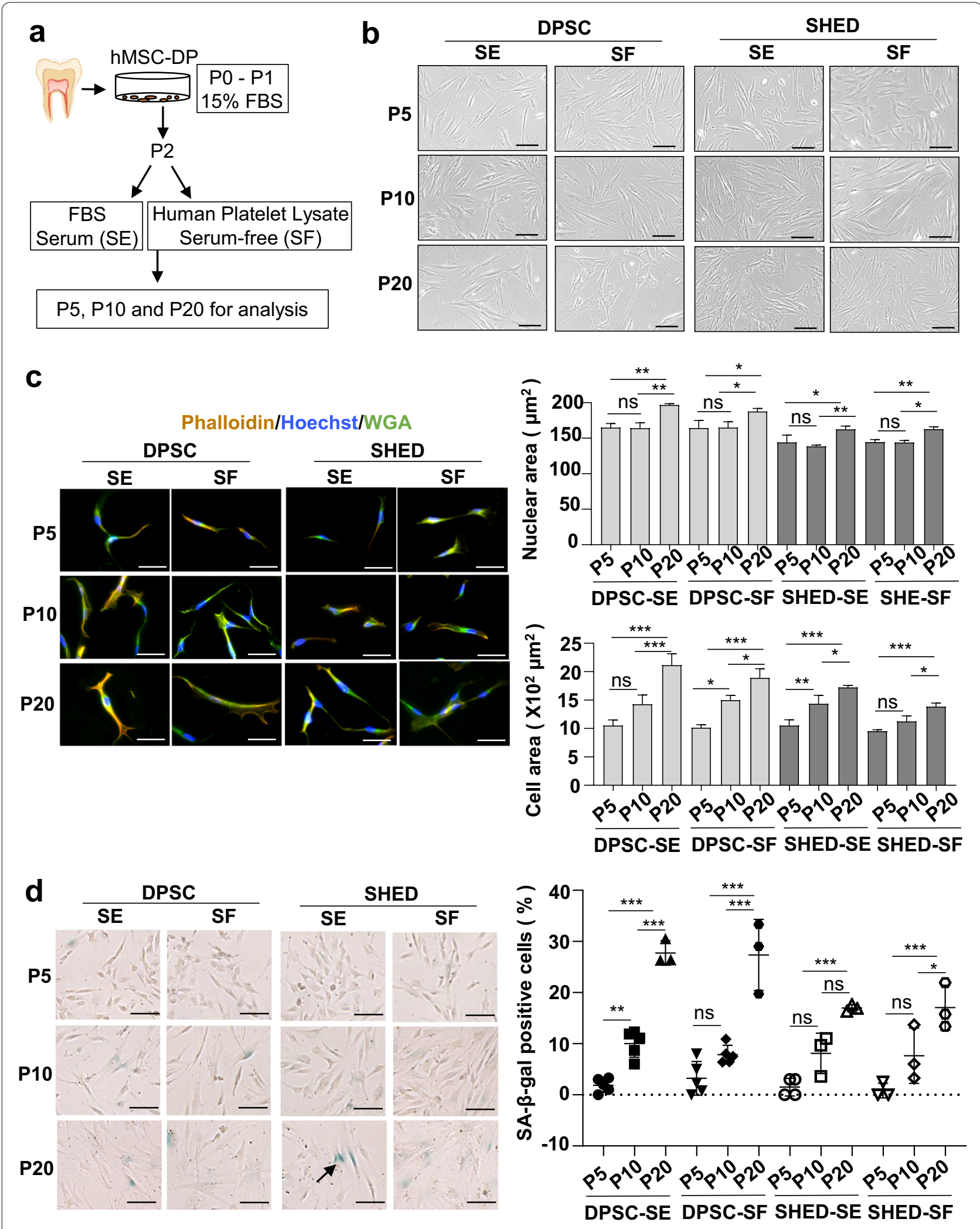

Fig. 1 (See legend on previous page.) 

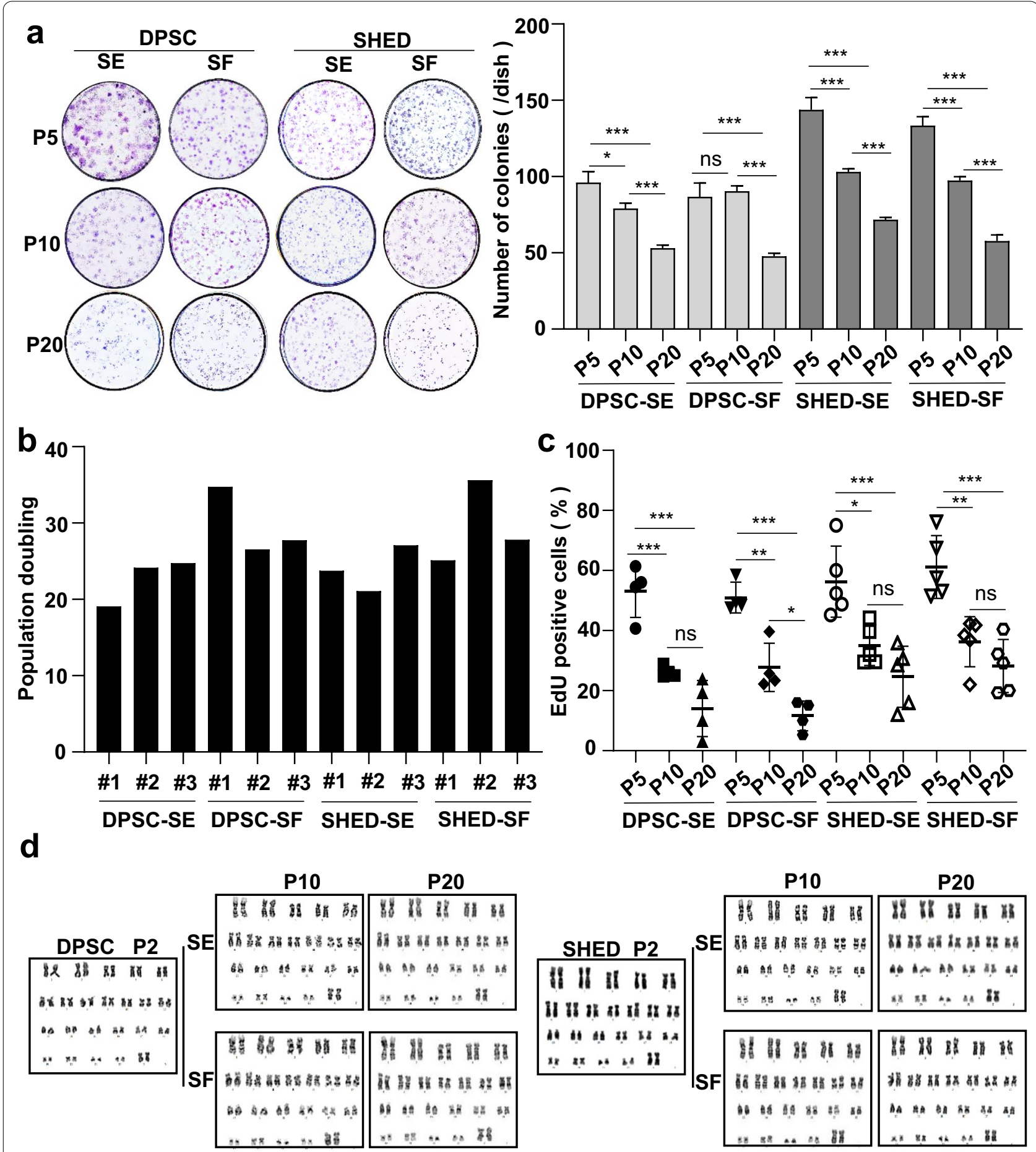

Fig. 2 Proliferation capacity and chromosomal stability of hMSC-DP at different passages. a Colony-forming ability of DPSCS and SHED under SE and SF culture conditions was assessed by CFU-F assays. The numbers of colonies at P5, P10 and P20 were calculated and compared. $n=3$ for each group. $\mathbf{b}$ Proliferation capacity of three groups of donor-derived DPSCs and three groups of donor-derived SHED was analyzed by population doubling assay. c Proliferation rate of hMSC-DP was assessed by EdU staining. The percentage of EdU-positive cells was calculated at P5, P10 and P20. d Chromosomal stability was assessed by karyotype analysis. The karyotypes of DPSCs and SHED at P5, P10 and P20 were examined. SE, serum; $\mathrm{SF}$, serum-free. Data shown as mean $\pm \mathrm{SEM} .{ }^{*} p<0.05,{ }^{* *} p<0.01,{ }^{* * *} p<0.001$. ns not significant 


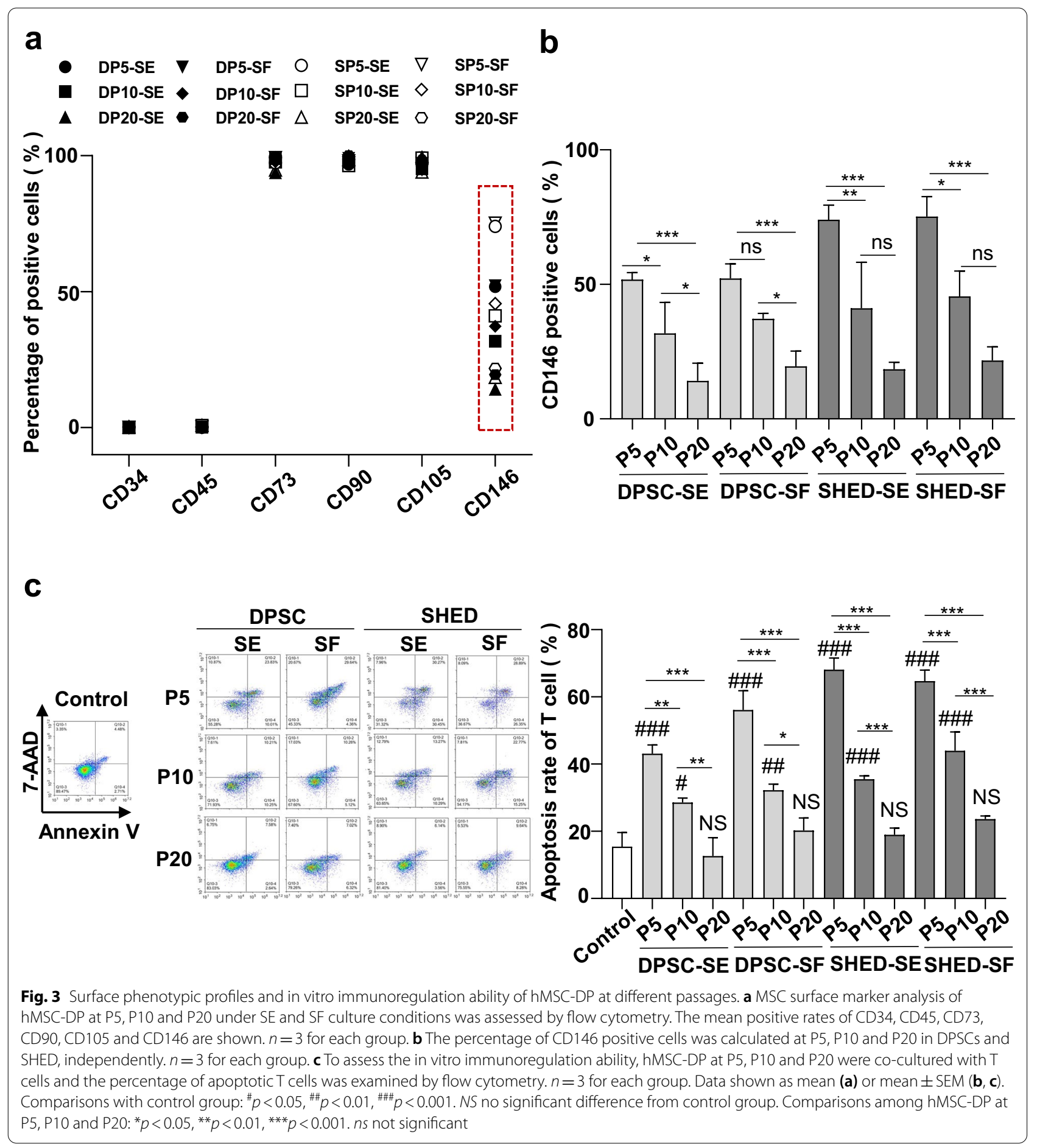

elevated immunomodulatory capability compared to SE-cultured hMSC-DP, though only DPSCs at P5 under SF culture conditions showed significantly elevated immune regulation capacity compared to SE-cultured DPSCs $(P<0.05)$ (Additional file 1 : Fig. S3c). These results indicate that hMSC-DP cultured in SF media may be more suitable for using in immunotherapy.

\section{Multilineage differentiation of hMSC-DP}

Multilineage differentiation potential is a critical parameter in evaluating the potency of MSCs. To assess the 
(See figure on next page.)

Fig. 4 Multilineage differentiation of hMSC-DP. a, b Osteogenic capacity of hMSC-DP. The percentage of alizarin red staining compared at P5, P10 and P20 (a). Western blot analysis showed the expression levels of osteogenic markers including Runx 2 and ALP (b). $n=3$ for each group. c, d Adipogenic capacity of hMSC-DP. The percentage of oil red o positive cells was calculated and analyzed at P5, P10 and P20 (c). Western blot analysis showed the expression levels of adipogenic markers including LPL and PPARY (d). $n=3$ for each group. e Neural differentiation of hMSC-DP. Immunofluorescent staining and western blot analysis showed the expression of neural markers $\beta$ III-tubulin and NeuN. Scale bar $=100 \mu m$. SE, serum; SF, serum-free. Data shown as mean $\pm \mathrm{SEM} .{ }^{*} p<0.05,{ }^{* *} p<0.01,{ }^{* * *} p<0.001$. ns not significant

osteogenic capacity of hMSC-DP, we cultured hMSCDP at P5, P10 and P20 in SE and SF osteogenic inductive media for two weeks. Alizarin red staining showed that hMSC-DP from P5 to P20 showed a tendency towards reduced mineralized nodule formation, indicating that continued passage impairs the osteogenic differentiation potential of hMSC-DP (Fig. 4a, Additional file 1: Fig. S4a). Western blot analysis confirmed that the continuous passage reduced the expression levels of runt-related transcription factor 2 (Runx2) and alkaline phosphatase (ALP) (Fig. 4b). Interestingly, SHED at P20 under SF culture conditions still possessed relatively strong osteogenic capacity, while DPSCs at P20 under SF culture conditions had a significantly elevated capacity to form mineralized nodules when compared to SE-cultured DPSCs (Additional file 1: Fig. S4a). All these results suggest that SF medium has advantages in maintaining the osteogenic capacity of hMSC-DP.

Our previous studies showed that hMSC-DP are capable of differentiating into adipocytes, but have a limited capacity to do so $[9,10]$. Here, we showed that hMSC-DP from P5 to P20 under SE or SF culture medium exhibited limited adipogenic differentiation capacity after 6 weeks of adipogenic induction (Fig. 4c, Additional file 1: Fig. S4b). Western blot analysis showed the expression levels of two adipocyte-specific transcripts, peroxisome proliferator-activated receptor- $\gamma 2$ (PPAR $\gamma$ ) and lipoprotein lipase (LPL), in each group (Fig. 4d).

Previous studies showed that DPSCs and SHED are capable of differentiating into neural cells, presumably due to their neural crest origin [37-39]. Here, we showed that hMSC-DP from P5 to P20 under SE or SF culture conditions maintain neurogenic differentiation capacity, as assessed by western blot and immunostaining to show expression of neuronal markers $\beta$ III tubulin and NeuN when cultured under neurogenic inductive conditions (Fig. 4e).

\section{hMSC-DP ameliorate Dextran sulfate sodium (DSS)-induced experimental colitis}

DSS-induced experimental colitis has been widely used to evaluate the in vivo immunomodulatory capacity of MSCs $[28,40]$. To further assess the immunomodulatory ability of hMSC-DP, we systemically infused SE and SFcultured hMSC-DP at P5, P10 and P20 into experimental colitis mice on day 3 after 3\% DSS induction. The experimental colitis mice were sacrificed at 8 days post DSS induction. On day 8, DPSC-SE at P5 and all DPSCs cultured under SF conditions improved the body weight of experimental colitis mice after intravenous infusion. DPSC-SF at P5 showed a superior capacity to rescue the body weight when compared to DPSC-SF at P10 $(P<0.05)$ and P20 $(P<0.01)$.

Intravenously infused SHED, except SHED-SF at P20, significantly elevated the body weight in DSS-induced colitis mice when compared to the DSS group. SHEDSF at P20 somewhat lost the ability to improve the body weight $(P<0.05)$ (Fig. 5a). Treatment with hMSC-DP at $\mathrm{P} 5$ resulted in a significantly improved disease activity index (DAI) compared to the untreated DSS group, whereas hMSC-DP at P20 failed to improve the DAI (Fig. 5b). Anatomical images of the colon of each group showed that DPSC-SE at P5 and all DPSCs under SF culture conditions had therapeutic effects for DSS-induced colitis, while SHED-SE at P5 and SHED-SF at P5 and P10 improved the colon length compared to the untreated DSS group (Fig. 5c, Additional file 1: Fig. S5b). Histological scores from HE staining images showed that hMSCDP from all groups, except DPSC-SE at P20 and SHED at P20, offered effective therapy for DSS-induced colitis (Fig. 5d, Additional file 1: Fig. S5c). In general, the immunoregulatory ability of stem cells decreased gradually with the continuous passage. The therapeutic effect of P10 hMSC-DP was weaker than that of P5 hMSC-DP in experimental colitis mice, while P20 hMSC-DP showed little therapeutic effect.

In general, culture conditions have little effect on the immunomodulatory capacity of SHED. However, SF-cultured DPSCs tend to exhibit a greater immunomodulatory effect than SE-cultured DPSCs, but the difference is not statistically significant (Additional file 1: Fig. S5).

\section{CD146 regulates the potency of hMSC-DP}

Previous studies showed that MSCs with high CD146 expression have superior osteogenic and immunoregulatory capacity relative to those with low CD146 expression [41-43]. We found that the expression level of CD146 in hMSC-DP decreased with passage (Fig. 6a). To further explore the role of CD146 in hMSC-DP, we blocked the expression of CD146 in hMSC-DP with siCD146 (Fig. 6b, 
a

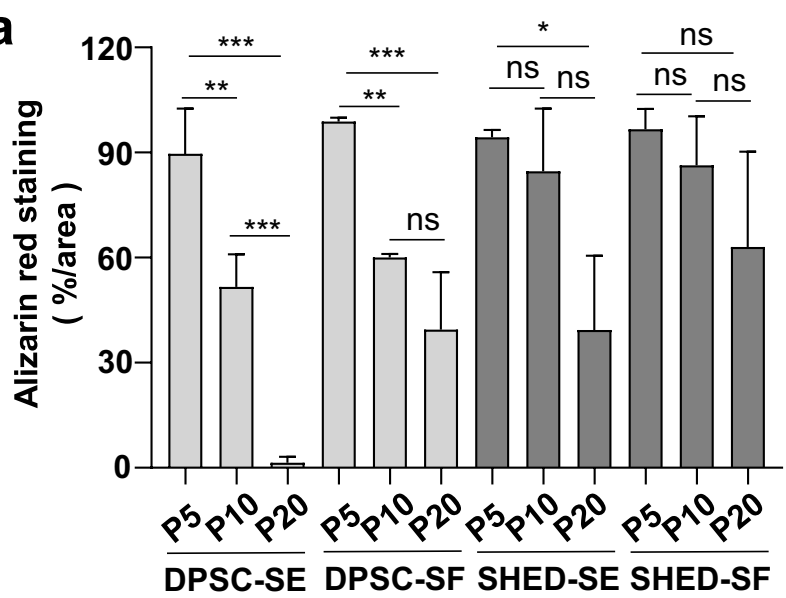

C

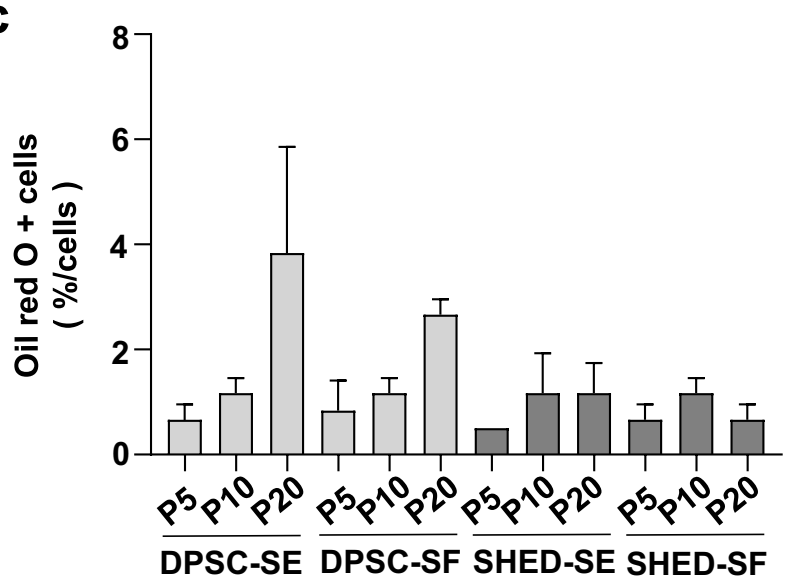

e
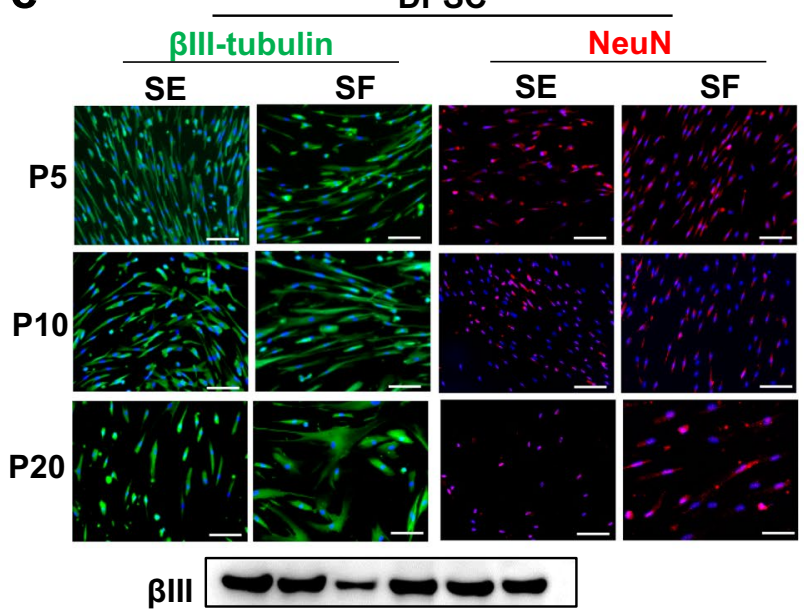

NeuN $-\infty$

GAPDH

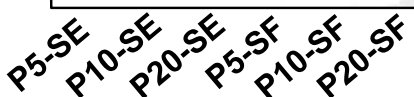

b

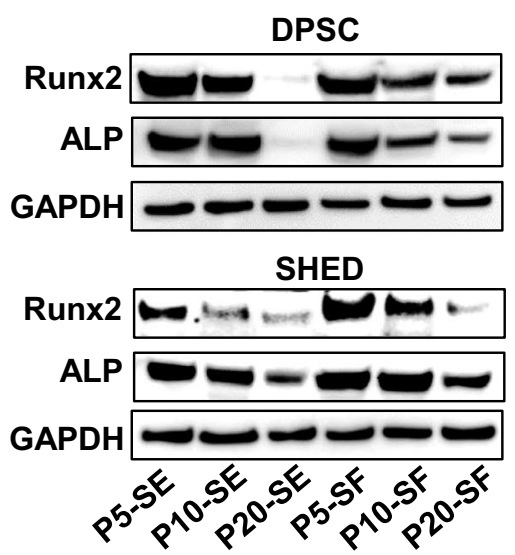

d

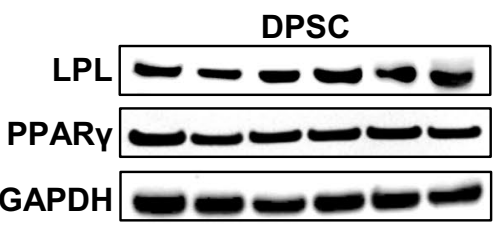

SHED

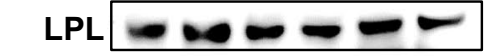

PPARY $\square-\infty$

GAPDH

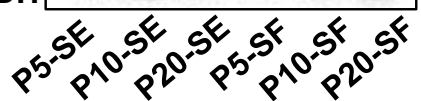

SHED
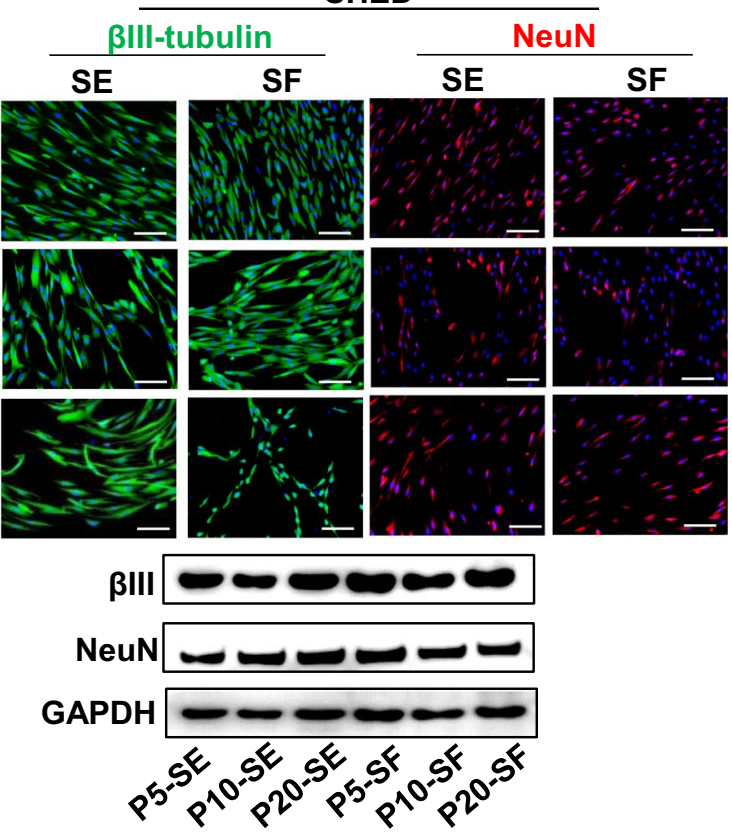

Fig. 4 (See legend on previous page.) 
(See figure on next page.)

Fig. 5 hMSC-DP ameliorate Dextran sulfate sodium (DSS)-induced experimental colitis. a Body weights were recorded every day for 8 days in DPSCs and SHED transplanted groups. The relative alteration of body weight was calculated. $n=3$ for each group. $\mathbf{b}$ Disease activity index (DAl) was evaluated on day 8 of DSS feeding. $n=3$ for each group. $\mathbf{c}$ Colon length was measured on day $8 . n=3$ for each group. $\mathbf{d}$ Histological score based on H\&E staining images was assessed on day 8 . The histological score of each group was calculated. $n=3$ for each group. SE, serum; SF, serum-free. Data shown as mean \pm SEM. Comparisons with untreated DSS group: ${ }^{\#} p<0.05,{ }^{\#} p<0.01,{ }^{\# \#} p<0.001$. NS not significantly different from DSS group. Comparisons of DPSCs or SHED at P5, P10 and P20: ${ }^{*} p<0.05,{ }^{* *} p<0.01,{ }^{* *} p<0.001$. ns not significant

c), as shown by western blot and flow cytometry. After siCD146 interference, the capacities for proliferation, in vitro immunoregulation and osteogenesis were significantly decreased, as indicated by EdU staining, apoptosis rate of $\mathrm{T}$ cells, alizarin red staining and western blot analysis of ALP and Runx2 (Fig. 6d-g). Furthermore, we analyzed the correlation between the potency of hMSCDP and the expression level of CD146 and found that the potency of hMSC-DP is positively correlated with the expression level of CD146, especially in proliferation, osteogenesis, and in vivo immunoregulatory capacity (Additional file 1: Fig. S6). These results suggest that CD146 is a functional surface molecule that represents the potency of hMSC-DP.

It is well-known that ERK/p-ERK pathway plays a critical role in regulating MSC proliferation and differentiation [27, 44, 45]. Here, we showed that the expression level of p-ERK, but not ERK, was consistent with the expression level of CD146 in hMSC-DP (Fig. 7a). Western blot results showed that siCD146 treatment downregulated the expression of p-ERK in hMSCDP (Fig. 7b), suggesting that CD146 may maintain the potency of hMSC-DP through ERK/p-ERK pathway. To test this hypothesis, we used PD98059, an ERK inhibitor, to reduce the expression of pERK in hMSC-DP, as assessed by western blot (Fig. 7c). ERK inhibitor treatment significantly reduced the proliferation rate and immunosuppression capacity of hMSC-DP, as assessed by EdU staining and $\mathrm{T}$ cell coculture assay (Fig. $7 \mathrm{~d}$, e). Interestingly, osteogenic differentiation was not affected in DPSCs group but elevated in SHED group after treated with a p-ERK inhibitor, as reported previously [27, 46]. These results indicate that CD146 may maintain hMSCDP proliferation and immune modulation capacities, but not osteogenic differentiation capacity, via ERK pathway.

\section{Discussion}

SHED and DPSCs possess elevated proliferation, differentiation and immunoregulation capacities [12, 13]. Recently, hMSC-DP have been used in clinics for tissue regeneration and immune therapies (Additional file 2: Table S1). Therefore, it is necessary to develop standard operation procedures (SOPs) for the clinical use of hMSC-DP. These SOPs should ultimately provide guidance and acceptance criteria for all necessary steps, such as clinical-grade cell preparation, transportation, biosafety tests, quality control and therapeutic methods [15]. Among these criteria, the quality and potency of hMSC-DP are critical for their therapeutic effects.

FBS has been generally used as an essential component for MSC culture [47-49]. However, it is becoming increasingly common to use serum-free culture to expand MSCs for clinical application [25, 47, 48]. Human platelet lysate shows benefits in promoting MSC proliferation and thus may serve as a possible alternative to replace FBS [47]. Here, we used human platelet lysatebased serum-free medium to culture hMSC-DP and found that this culture medium is appropriate for expansion of hMSC-DP in terms of achieving normal morphology, cell proliferation, multipotency, immunoregulation, surface molecule expression, and karyotypes.

MSCs can be derived from multiple tissues, but MSCs from different tissue sources may possess unique characteristics [49]. hMSC-DP are derived from neural crest cells and easily differentiate into neural cells [37]. Here, we further reveal that hMSC-DP maintain neural differentiation potential even at passage 20. Additionally, we show that hMSC-DP have osteogenic differentiation potential and maintain their osteogenic capacity even after continuous passage in SF culture conditions. As dental pulp-derived MSCs, hMSC-DP possess unique characteristics including elevated proliferation and the potential for neural and odonto/osteo-differentiation $[9,10,50]$. Accurate evaluation of hMSC-DP characteristics can help to ensure their quality for future clinical application.

MSC-mediated immunomodulation and immune therapies have been widely reported [51-53]. The MSC Committee of the ISCT published a working proposal for the immunological characterization of MSCs in 2013, including MSC immunophenotyping, an in vitro cell responder assay and in vivo animal models [54]. In this study, we found that hMSC-DP at early passages such as P5 show optimal immunoregulation effects in vitro in a $\mathrm{T}$ cell coculture system and in vivo in a DSS-induced colitis mouse model. However, continued passaging (to P10 and beyond) reduces their therapeutic capacity for colitis mice, which may relate to their diminished ability to induce $\mathrm{T}$ cell apoptosis [28]. 

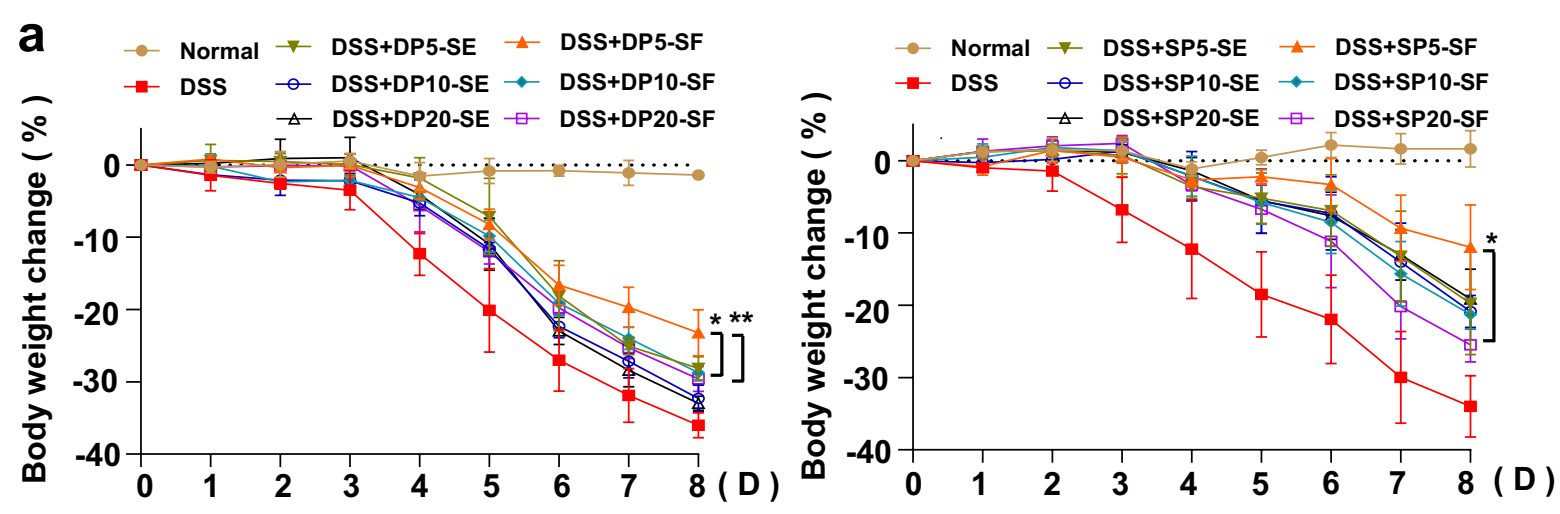

b
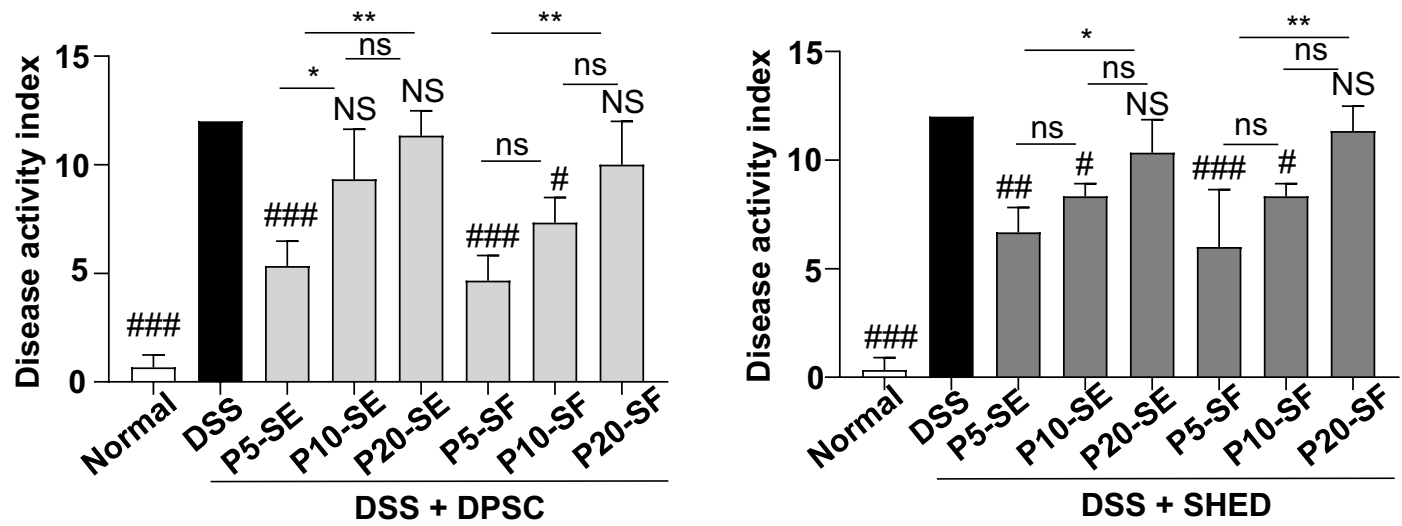

C
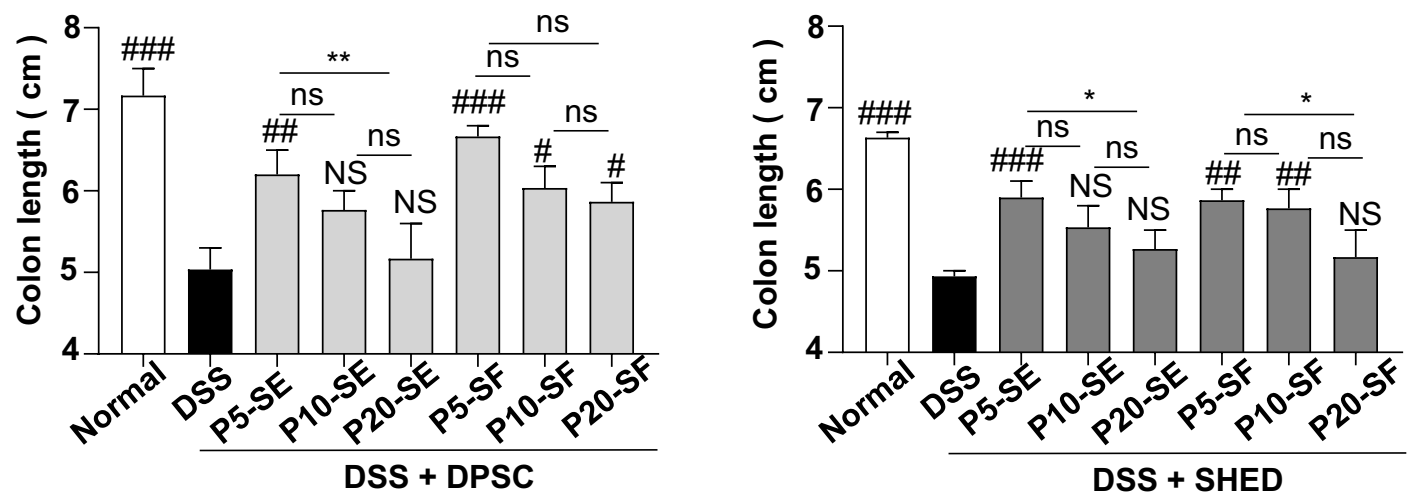

d

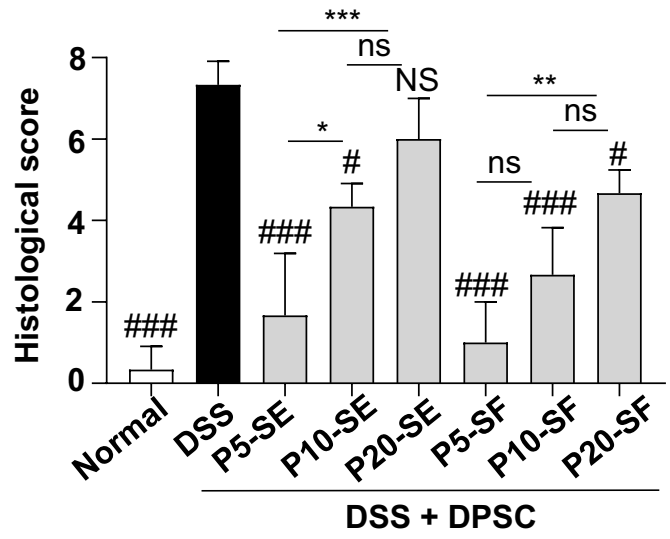

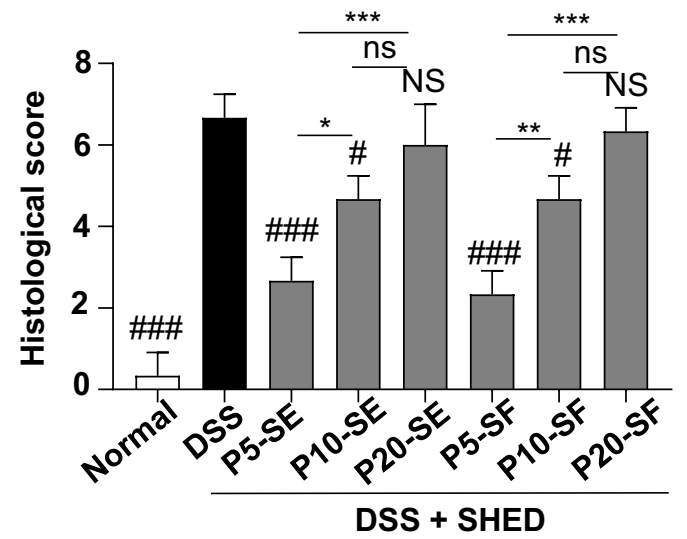

Fig. 5 (See legend on previous page.) 

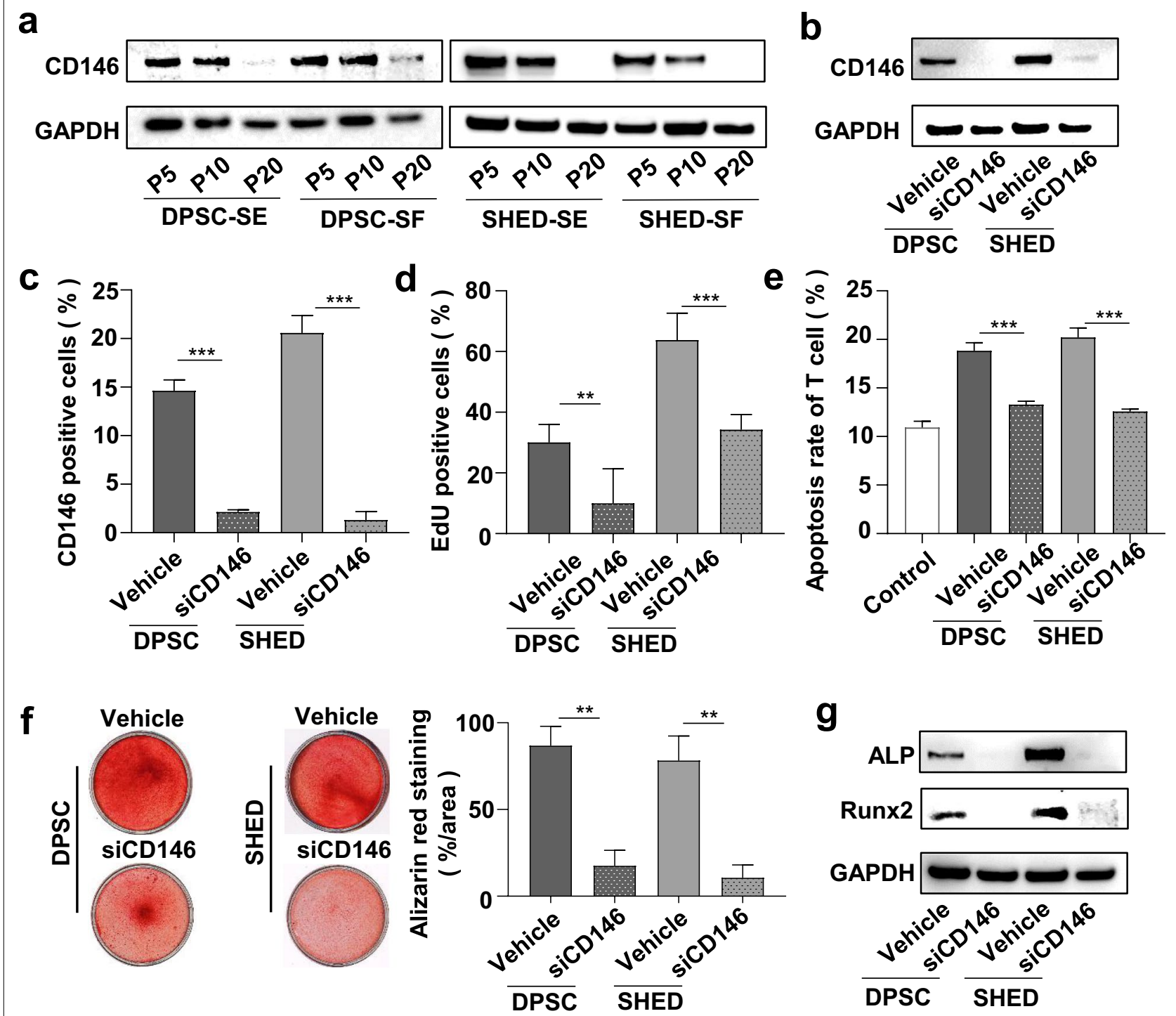

Fig. 6 CD146 expression is associated with hMSC-DP proliferation, differentiation and immune modulation. a Western blot analysis showed the expression of CD146 in DPSCs and SHED at P5, P10 and P20 under SE or SF culture conditions. b The expression level of CD146 in DPSCs and SHED at P10 was downregulated by siCD146, as assessed by western blot. c Flow cytometry analysis showed the percentage of CD146-positive cells was reduced after siCD146 treatment. $n=3$ for each group. $\mathbf{d}$ EdU staining showed the proliferation rate of hMSC-DP was decreased after siCD146 treatment. $n=3$ for each group. e After co-culturing siCD146-transfected hMSC-DP with T cells, the percentage of apoptotic T cells was reduced, as assessed by flow cytometry. $n=3$ for each group. $\mathbf{f}, \mathbf{g}$ Osteogenesis of hMSC-DP was impaired by knocking down CD146, as assessed by the percentage of alizarin red staining $(\mathbf{f})$ and western blot of osteogenic markers $(\mathbf{g}) . n=3$ for each group. SE, serum; SF, serum-free. Data shown as mean \pm SEM. ${ }^{* *} p<0.01,{ }^{* * *} p<0.001$. ns not significant

CD146 was initially identified as a specific marker of malignant melanoma [55]. Previous studies showed that CD146 is expressed on the surface of human bone marrow MSCs, human umbilical cord-derived MSCs, human adipose tissue-derived MSCs, DPSCs and SHED [36, 42, 56-58]. MSCs with high expression of CD146 show elevated osteogenic and immunoregulatory abilities compared to those with low CD146 expression [41,
$42,56]$. Here, we found that the expression levels of traditional MSC surface markers, including CD73, CD90 and CD105, fail to reflect the potency of hMSC-DP, while the expression level of CD146 correlates with hMSC-DP capacity for proliferation, differentiation, and immunoregulation $[26,59]$. Therefore, we propose CD146 as a functional surface molecule to predict the quality of hMSC-DP. 


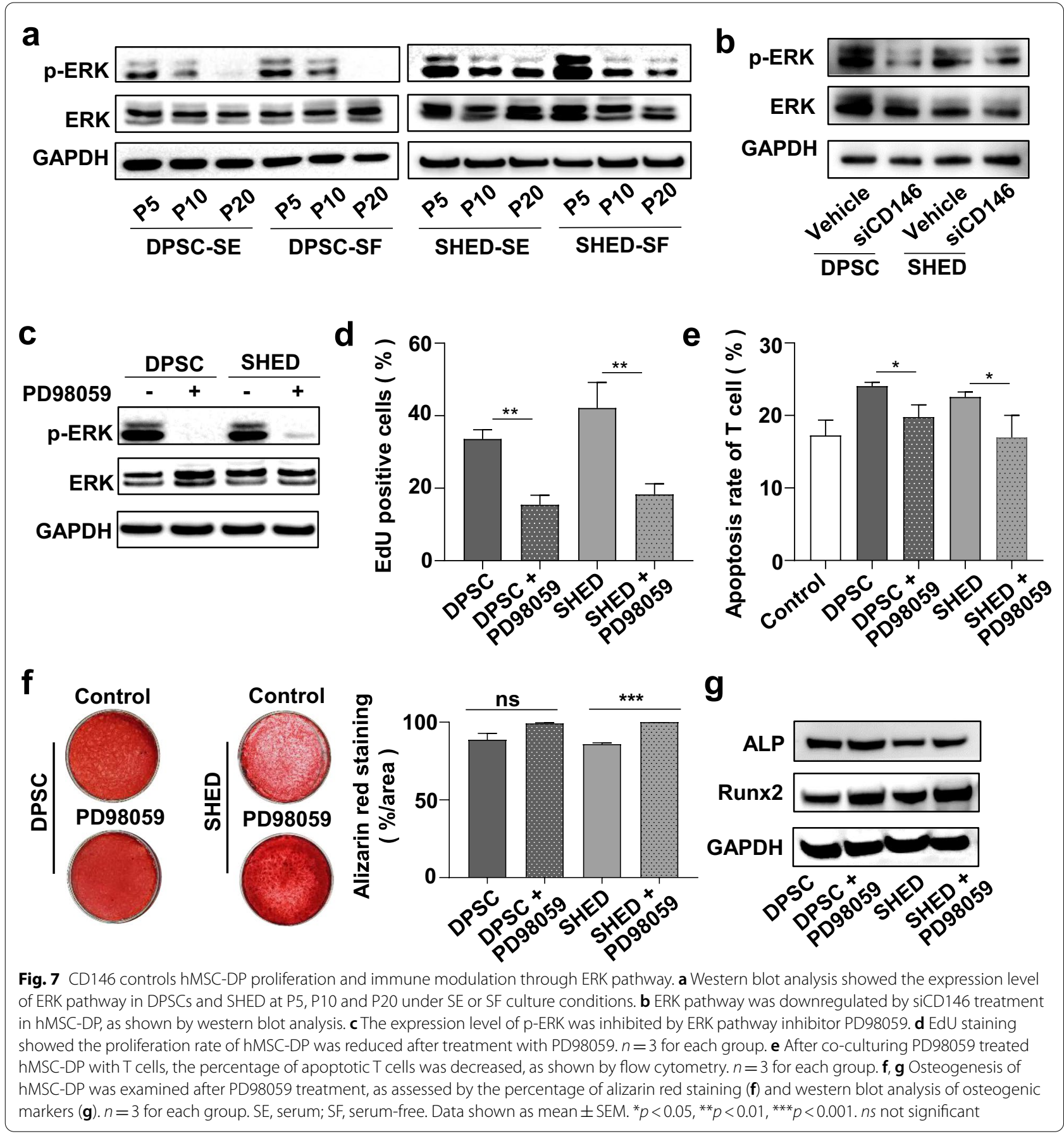

ERK/p-ERK pathway plays a critical role in regulating proliferation, immunoregulation and differentiation of MSCs [27, 60, 61]. Previous studies showed that CD146 expression is associated with the activation of ERK pathway during epithelial-mesenchymal transition [62] and tumor angiogenesis [63]. In this study, we demonstrated that CD146 maintained cell proliferation and immunomodulation through ERK/p-ERK pathway, but not osteogenic differentiation. Usually, a highly proliferative state is incompatible with differentiation in MSCs. The controversial role of ERK signaling has been discussed in the context of osteogenic differentiation of MSCs [45]. Activation of ERK signaling in human bone marrowderived MSCs promotes osteogenic differentiation [64, 65], while upregulation of ERK/p-ERK pathway contributes to the suppression of osteogenesis of MSCs [27, 44, 
46]. ERK/p-ERK may regulate other pathways, such as PI3-kinase/Akt or P38 pathway, to affect osteogenic differentiation of hMSC-DP [66, 67].

The minimal criteria for defining MSCs proposed by ISCT are quite basic and general [8], but it may fail to totally reflect the comprehensive characteristics of MSCs, such as their trophic activity [68] and immunomodulatory capacity [28]. Therefore, it may be insufficient to predict the potency of MSCs for clinical applications. Moreover, different tissue-derived MSCs may exhibit parent tissue specificity and possess different functional potential [69]. With the improvement of our understanding of the functional and tissue-specific characteristics of MSCs, it is necessary to define the criteria of tissuespecific MSCs for translational precision therapies. To meet upcoming requirements for defining optimal MSCs for clinical application, we propose additional criteria to define the potency of hMSC-DP: (1) Adherence to plastic forming CFU-F in serum-free culture conditions; (2) CD146 expressed by over 30\% of cells; (3) Neural differentiation potential. These added criteria aim for standardized identification of hMSC-DP for clinical use. In our study, we found that $10-80 \%$ of SHED and $5-70 \%$ of DPSCs expressed CD146, which was positively correlated with stem cell function. Some critical capacities of hMSC-DP were significantly decreased with the reduced expression of CD146 at passage 10. The positive rate of CD146 expression detected by flow cytometry was about $30-40 \%$ at passage 10 in our study. Therefore, we propose that CD146 can serve as a functional surface molecule to evaluate the potency of hMSC-DP. When CD146 positive rate is above $30 \%$, hMSC-DP can provide optimal therapeutic effect in DDS-induced colitis mouse model.

\section{Conclusion}

We explored the physiological and functional status of hMSC-DP in the SF culture system and established minimal criteria to identify the potency of hMSC-DP for potential clinical application. CD146 is a functional surface molecule that reflects the potency of hMSC-DP.

\footnotetext{
Abbreviations

hMSC-DP: Human mesenchymal stem cells from dental pulp; DPSCs: Postnatal human dental pulp stem cells; SHED: Stem cells from human exfoliated deciduous teeth; SE: Serum; SF: Serum-free; MSCs: Mesenchymal stem cells; ISCT: International Society for Cellular Therapy; SOPs: Standard operation procedures; FBS: Fetal bovine serum; EdU: 5-Ethynyl-20-deoxyuridine; PD: Population doublings; CFU: Colony-forming unit; WGA: Wheat germ agglutinin; $\beta$-gal: $\beta$-Galactosidase; SA- $\beta$-gal: Senescence-associated $\beta$-galactosidase; Runx2: Runt-related transcription factor 2; ALP: Alkaline phosphatase; PPARY: Peroxisome proliferator-activated receptor- $\gamma 2$; LPL: Lipoprotein lipase; DSS: Dextran Sulfate Sodium; DAl: Disease activity index; HAl: Histological activity index.
}

\section{Supplementary Information}

The online version contains supplementary material available at https://doi. org/10.1186/s13287-021-02559-4.

Additional file 1: Fig. S1 Characterization of the morphology of hMSCDP under SF culture conditions. a-b Cell morphology analysis by highcontent imaging. The nuclear area (a) and cell area (b) of DPSCs and SHED are shown in SE and SF culture conditions. $n=3$ for each group. c Cellular senescence assay. The percentage of SA- $\beta$-gal positive cells was calculated and compared between SE and SF culture conditions. $n=3 \sim 5$ for each group. SE, serum; SF, serum-free. Data shown as mean \pm SEM. ns, not significant. Fig. S2 Proliferation capacity of hMSC-DP under SF culture conditions. a CFU-F assay. The numbers of colonies of hMSC-DP under SE or SF culture conditions were calculated and compared. $n=3$ for each group. b Population doubling scores were calculated and compared between SE and SF culture conditions in DPSCS and SHED groups, independently. $\mathrm{n}=3$ for each group. $\mathbf{c}$ EdU assay. The percentage of EdU-positive cells were calculated and compared between SE and SF culture conditions in DPSCs and SHED groups, independently. $n=3$ for each group. Scale bar $=200 \mu \mathrm{m}$. SE, serum; SF, serum-free. Data shown as mean \pm SEM ${ }^{*} p<0.05$. ns, not significant. Fig. S3 Surface phenotypic profiles and in vitro immunoregulation ability of hMSC-DP. $\mathbf{a}-\mathbf{b}$ Flow cytometry showed the percentage of CD146-positive hMSC-DP under SE or SF culture conditions at P5, P10 and P20 (a). The percentage of CD146-positive cells was compared between SE and SF culture conditions (b). $n=3$ for each group. c The percentage of apoptotic T cells was calculated in SE and SF culture conditions. $n=3$ for each group. SE, serum; SF, serum-free. Data shown as mean \pm SEM. ${ }^{*} p<0.05$. ns, not significant. Fig. S4 Multilineage differentiation of hMSC-DP. a Alizarin red staining assay. The osteogenic capacity was compared between SE and SF culture conditions at P5, P10 and P20. $n=3$ for each group. $\mathbf{b}$ Oil red $O$ staining assay. The adipogenic capacity of hMSC-DP was compared between SE and SF culture conditions at P5, P10 and P20. n=3 for each group. Scale bar $=50 \mu \mathrm{m}$. $\mathrm{SE}$, serum; SF, serum-free. Data shown as mean $\pm \mathrm{SEM}$. ${ }^{* *} p<0.01$. ns, not significant. Fig. S5 Therapeutic effects of hMSC-DP on experimental colitis. a Disease activity index (DAI) of DPSC- and SHED-treated groups was compared between SE and SF culture conditions at P5, P10 and P20. $n=3$ for each group. $\mathbf{b}$ The colon length after DPSCs and SHED treatment was compared between SE and SF culture conditions at P5, P10 and P20. n=3 for each group. $\mathbf{c}$ Histological structure was examined by H\&E staining. The histological score of each group was compared between SE and SF culture conditions. $n=3$ for each group. Scale bar $=100 \mu \mathrm{m}$. SE, serum; SF, serum-free. Data shown as mean \pm SEM. ns, not significant. Fig. S6 The expression level of CD146 is associated with hMSC-DP properties. a The correlation of CD146 with experimental parameters. $\mathbf{b}$ Linear regression plots showed the correlation between CD146 expression and experimental parameters including CFU-F clones, percentage of EdU positive cells, percentage of Alizarin red positive area, percentage of SA- $\beta$-gal positive cells, DAl scores, HAl scores and colon length.

Additional file 2. Table S1. Summary of clinical trials of hMSC-DP.

\section{Acknowledgements}

We thank Mr. Lulu (South China Center of Craniofacial Stem Cell Research, Sun Yat-Sen University) for his kind help in this study.

\section{Authors' contributions}

$\mathrm{LM}$ and $\mathrm{ZH}$ contributed to designing the study plan, performing experimental procedures and drafting the manuscript. DW contributed to data acquisition, analysis and interpretation. XK and XM contributed to data analysis and interpretation. SS contributed to the project conception, experimental design, writing manuscript and supervision. All authors approved the final version of the manuscript.

\section{Funding}

This study was supported by grants from the National Natural Science Foundation of China (81700928 to L.M.), the Youth Teacher Training Project of Sun Yat-sen University (17ykpy71 to L.M.), the Pearl River Talent Recruitment Program (2019ZT08Y485 and 2019QN01Y138 to S.S., 2019JC01Y182 to X.K.), 
the Guangdong Financial Fund for High-Caliber Hospital Construction (1742018-XMZC-0001-03-0125, D-07 to S.S., D-11 to X.K.), the National Science and Technology Major Project of the Ministry of Science and Technology of China (2018ZX10302207), the Sun Yat-sen University Young Teacher Key Cultivation Project (18ykzd05 to X.K.) and the Natural Science Foundation of Guangdong (2016A030313262 to X.M.).

\section{Availability of data and materials}

All data generated or analyzed during this study are included in this published article and its Additional files.

\section{Declarations}

\section{Ethics approval and consent to participate}

Human samples collection was approved by the Medical Ethics Committee of Hospital of Stomatology, Sun Yat-sen University (Protocol Number: KQEC2020-055-01). All animal experiments were performed under an institutionally approved protocol for the use of animal research of Sun Yat-sen University (Protocol Number: SYSU-IACUC-2020-000394).

\section{Consent for publication}

Not applicable.

\section{Competing interests}

The authors declare that they have no competing interests.

Received: 16 April 2021 Accepted: 16 Auqust 2021

Published online: 30 August 2021

\section{References}

1. Pittenger MF, Discher DE, Péault BM, et al. Mesenchymal stem cell perspective: cell biology to clinical progress. NPJ Regen Med. 2019;4:22.

2. Matthay MA, Calfee CS, Zhuo H, et al. Treatment with allogeneic mesenchymal stromal cells for moderate to severe acute respiratory distress syndrome (START study): a randomised phase 2a safety trial. Lancet Respir Med. 2019;7(2):154-62.

3. Meng F, Xu R, Wang S, et al. Human umbilical cord-derived mesenchymal stem cell therapy in patients with COVID-19: a phase 1 clinical trial. Signal Transduct Target Ther. 2020;5(1):172.

4. Xu X, Jiang W, Chen L, et al. Evaluation of the safety and efficacy of using human menstrual blood-derived mesenchymal stromal cells in treating severe and critically ill COVID-19 patients: an exploratory clinical trial. Clin Transl Med. 2021;11(2):e297.

5. Marquez-Curtis LA, Janowska-Wieczorek A, McGann LE, et al. Mesenchymal stromal cells derived from various tissues: biological, clinical and cryopreservation aspects. Cryobiology. 2015;71(2):181-97.

6. Kozlowska U, Krawczenko A, Futoma K, et al. Similarities and differences between mesenchymal stem/progenitor cells derived from various human tissues. World J Stem Cells. 2019;11(6):347-74.

7. Ullah I, Subbarao RB, Rho GJ. Human mesenchymal stem cells: current trends and future prospective. Biosci Rep. 2015;35(2):e00191.

8. Dominici M, Le Blanc K, Mueller I, et al. Minimal criteria for defining multipotent mesenchymal stromal cells. The international society for cellular therapy position statement. Cytotherapy. 2006;8(4):315-7.

9. Gronthos S, Mankani M, Brahim J, et al. Postnatal human dental pulp stem cells (DPSCs) in vitro and in vivo. Proc Natl Acad Sci USA. 2000;97(25):13625-30.

10. Miura M, Gronthos S, Zhao M, et al. SHED: stem cells from human exfoliated deciduous teeth. Proc Natl Acad Sci USA. 2003;100(10):5807-12.

11. Botelho J, Cavacas MA, Machado V, et al. Dental stem cells: recent progresses in tissue engineering and regenerative medicine. Ann Med. 2017:49(8):644-51.

12. Shi $X$, Mao J, Liu Y. Pulp stem cells derived from human permanent and deciduous teeth: biological characteristics and therapeutic applications. Stem Cells Transl Med. 2020;9(4):445-64.

13. Sui $B$, Wu $D$, Xiang $L$, et al. Dental pulp stem cells: from discovery to clinical application. J Endod. 2020;46(9):S46-55.
14. Bakopoulou A, About I. Stem cells of dental origin: current research trends and key milestones towards clinical application. Stem Cells Int. 2016:2016:4209891.

15. Yamada Y, Nakamura-Yamada S, Kusano K, et al. Clinical potential and current progress of dental pulp stem cells for various systemic diseases in regenerative medicine: a concise review. Int J Mol Sci. 2019;20(5):1132.

16. Xuan K, Li B, Guo H, et al. Deciduous autologous tooth stem cells regenerate dental pulp after implantation into injured teeth. Sci Transl Med. 2018;10(455):eaaf3227.

17. Prasad MGS, Ramakrishna J, Babu DN. Allogeneic stem cells derived from human exfoliated deciduous teeth (SHED) for the management of periapical lesions in permanent teeth: two case reports of a novel biologic alternative treatment. J Dent Res Dent Clin Dent Prospects. 2017;11(2):117-22.

18. Manimaran K, Sankaranarayanan S, Ravi VR, et al. Treatment of osteoradionecrosis of mandible with bone marrow concentrate and with dental pulp stem cells. Ann Maxillofac Surg. 2014;4(2):189-92.

19. Ankrum J, Karp JM. Mesenchymal stem cell therapy: two steps forward, one step back. Trends Mol Med. 2010;16(5):203-9.

20. Jin X, Xu Q, Champion K, et al. Endotoxin contamination of apolipoprotein A-l: effect on macrophage proliferation-a cautionary tale. Atherosclerosis. 2015;240(1):121-4.

21. Tekkatte C, Gunasingh GP, Cherian KM, et al. "Humanized" stem cell culture techniques: the animal serum controversy. Stem Cells Int. 2011;2011:504723.

22. Spees JL, Gregory CA, Singh $H$, et al. Internalized antigens must be removed to prepare hypoimmunogenic mesenchymal stem cells for cell and gene therapy. Mol Ther. 2004;9(5):747-56.

23. Coates DE, Alansary M, Friedlander $L$, et al. Dental pulp stem cells in serum-free medium for regenerative medicine. J R Soc N Z. 2019;50(1):80-90.

24. Qu C, Brohlin M, Kingham PJ, et al. Evaluation of growth, stemness, and angiogenic properties of dental pulp stem cells cultured in cGMP xeno-/ serum-free medium. Cell Tissue Res. 2020;380(1):93-105.

25. Iwanaka T, Yamaza T, Sonoda S, et al. A model study for the manufacture and validation of clinical-grade deciduous dental pulp stem cells for chronic liver fibrosis treatment. Stem Cell Res Ther. 2020;11(1):134.

26. Matsui M, Kobayashi T, Tsutsui TW. CD146 positive human dental pulp stem cells promote regeneration of dentin/pulp-like structures. Hum Cell. 2018;31(2):127-38.

27. Liu $Y$, Jing $H$, Kou $X$, et al. PD-1 is required to maintain stem cell properties in human dental pulp stem cells. Cell Death Differ. 2018;25(7):1350-60.

28. Akiyama K, Chen C, Wang D, et al. Mesenchymal-stem-cell-induced immunoregulation involves FAS-ligand-/FAS-mediated T cell apoptosis. Cell Stem Cell. 2012;10(5):544-55.

29. Haasters F, Prall WC, Anz D, et al. Morphological and immunocytochemical characteristics indicate the yield of early progenitors and represent a quality control for human mesenchymal stem cell culturing. J Anat. 2009;214(5):759-67.

30. Bray M-A, Singh S, Han H, et al. Cell Painting, a high-content image-based assay for morphological profiling using multiplexed fluorescent dyes. Nat Protoc. 2016;11(9):1757-74.

31. Zhao H, Darzynkiewicz Z. Biomarkers of cell senescence assessed by imaging cytometry. Methods Mol Biol. 2013;965:83-92.

32. Colter DC, Sekiya I, Prockop DJ. Identification of a subpopulation of rapidly self-renewing and multipotential adult stem cells in colonies of human marrow stromal cells. Proc Natl Acad Sci USA. 2001;98(14):7841-5.

33. Bernardo ME, Zaffaroni N, Novara F, et al. Human bone marrow derived mesenchymal stem cells do not undergo transformation after long-term in vitro culture and do not exhibit telomere maintenance mechanisms. Cancer Res. 2007;67(19):9142-9.

34. Lange C, Cakiroglu F, Spiess AN, et al. Accelerated and safe expansion of human mesenchymal stromal cells in animal serum-free medium for transplantation and regenerative medicine. J Cell Physiol. 2007;213(1):18-26.

35. Wang Z, Xu Q, Zhang N, et al. CD146, from a melanoma cell adhesion molecule to a signaling receptor. Signal Transduct Target Ther. 2020;5(1):148.

36. Shi S, Gronthos S. Perivascular niche of postnatal mesenchymal stem cells in human bone marrow and dental pulp. J Bone Miner Res. 2003;18(4):696-704. 
37. Mayo V, Sawatari Y, Huang CY, et al. Neural crest-derived dental stem cells—where we are and where we are going. J Dent. 2014;42(9):1043-51.

38. Li D, Zou X-Y, El-Ayachi I, et al. Human dental pulp stem cells and gingival mesenchymal stem cells display action potential capacity in vitro after neuronogenic differentiation. Stem Cell Rev Rep. 2019;15(1):67-81.

39. Anoop M, Datta I. Stem cells derived from human exfoliated deciduous teeth (shed) in neuronal disorders: a review. Curr Stem Cell Res Ther. 2021;16(5):535-50.

40. Shuai Y, Liao L, Su X, et al. Melatonin treatment improves mesenchymal stem cells therapy by preserving stemness during long-term in vitro expansion. Theranostics. 2016;6(11):1899-917.

41. Wangler S, Menzel U, Li Z, et al. CD146/MCAM distinguishes stem cell subpopulations with distinct migration and regenerative potential in degenerative intervertebral discs. Osteoarthr Cartil. 2019;27(7):1094-105.

42. Wu CC, Liu FL, Sytwu HK, et al. CD146+ mesenchymal stem cells display greater therapeutic potential than CD146-cells for treating collageninduced arthritis in mice. Stem Cell Res Ther. 2016;7:23.

43. Samsonraj RM, Rai B, Sathiyanathan $P$, et al. Establishing criteria for human mesenchymal stem cell potency. Stem Cells. 2015;33(6):1878-91.

44. Higuchi $\mathrm{C}$, Myoui $\mathrm{A}$, Hashimoto $\mathrm{N}$, et al. Continuous inhibition of MAPK signaling promotes the early osteoblastic differentiation and mineralization of the extracellular matrix. J Bone Miner Res. 2002;17(10):1785-94.

45. Schindeler A, Little DG. Ras-MAPK signaling in osteogenic differentiation: friend or foe? J Bone Miner Res. 2006;21(9):1331-8.

46. Li B, Qu C, Chen C, et al. Basic fibroblast growth factor inhibits osteogenic differentiation of stem cells from human exfoliated deciduous teeth through ERK signaling. Oral Dis. 2012;18(3):285-92.

47. Astori G, Amati E, Bambi F, et al. Platelet lysate as a substitute for animal serum for the ex-vivo expansion of mesenchymal stem/stromal cells: present and future. Stem Cell Res Ther. 2016;7(1):93.

48. Capelli C, Pedrini O, Valgardsdottir R, et al. Clinical grade expansion of MSCs. Immunol Lett. 2015;168(2):222-7.

49. Paliwal S, Chaudhuri R, Agrawal A, et al. Human tissue-specific MSCs demonstrate differential mitochondria transfer abilities that may determine their regenerative abilities. Stem Cell Res Ther. 2018;9(1):298.

50. Ma L, Makino Y, Yamaza H, et al. Cryopreserved dental pulp tissues of exfoliated deciduous teeth is a feasible stem cell resource for regenerative medicine. PLoS ONE. 2012;7(12):e51777.

51. Andrukhov O, Behm C, Blufstein A, et al. Immunomodulatory properties of dental tissue-derived mesenchymal stem cells: implication in disease and tissue regeneration. World J Stem Cells. 2019;11(9):604-17.

52. Li N, Hua J. Interactions between mesenchymal stem cells and the immune system. Cell Mol Life Sci. 2017;74(13):2345-60.

53. Shi Y, Wang Y, Li Q, et al. Immunoregulatory mechanisms of mesenchymal stem and stromal cells in inflammatory diseases. Nat Rev Nephrol. 2018;14(8):493-507.

54. Krampera M, Galipeau J, Shi Y, et al. Immunological characterization of multipotent mesenchymal stromal cells - the international society for cellular therapy (ISCT) working proposal. Cytotherapy. 2013;15(9):1054-61.
55. Wang Z, Xu Q, Zhang N, et al. CD146, from a melanoma cell adhesion molecule to a signaling receptor. Signal Transduct Target Ther. 2020:5(1):1-5.

56. Bowles AC, Kouroupis D, Willman MA, et al. Signature quality attributes of CD146(+) mesenchymal stem/stromal cells correlate with high therapeutic and secretory potency. Stem Cells. 2020;38(8):1034-49.

57. Li X, Guo W, Zha K, et al. Enrichment of CD146(+) adipose-derived stem cells in combination with articular cartilage extracellular matrix scaffold promotes cartilage regeneration. Theranostics. 2019;9(17):5105-21.

58. Yamaza T, Kentaro A, Chen C, et al. Immunomodulatory properties of stem cells from human exfoliated deciduous teeth. Stem Cell Res Ther. 2010;1(1):5.

59. Wang XT, Rao NQ, Fang TJ, et al. Comparison of the properties of CD146 positive and CD146 negative subpopulations of stem cells from human exfoliated deciduous teeth. Beijing Da Xue Xue Bao Yi Xue Ban. 2018:50(2):284-92.

60. Almalki SG, Agrawal DK. ERK signaling is required for VEGF-ANEGFR2induced differentiation of porcine adipose-derived mesenchymal stem cells into endothelial cells. Stem Cell Res Ther. 2017;8(1):113.

61. Zhang S, Chuah SJ, Lai RC, et al. MSC exosomes mediate cartilage repair by enhancing proliferation, attenuating apoptosis and modulating immune reactivity. Biomaterials. 2018;156:16-27.

62. Ma Y, Zhang H, Xiong C, et al. CD146 mediates an E-cadherin-to-Ncadherin switch during TGF- $\beta$ signaling-induced epithelial-mesenchymal transition. Cancer Lett. 2018;430:201-14.

63. Jiang T, Zhuang J, Duan H, et al. CD146 is a coreceptor for VEGFR-2 in tumor angiogenesis. Blood. 2012;120(11):2330-9.

64. Jaiswal RK, Jaiswal N, Bruder SP, et al. Adult human mesenchymal stem cell differentiation to the osteogenic or adipogenic lineage is regulated by mitogen-activated protein kinase. J Biol Chem. 2000;275(13):9645-52.

65. Murakami J, Ishii M, Suehiro F, et al. Vascular endothelial growth factor-C induces osteogenic differentiation of human mesenchymal stem cells through the ERK and RUNX2 pathway. Biochem Biophys Res Commun. 2017;484(3):710-8.

66. Baker N, Sohn J, Tuan RS. Promotion of human mesenchymal stem cell osteogenesis by PI3-kinase/Akt signaling, and the influence of caveolin-1/cholesterol homeostasis. Stem Cell Res Ther. 2015;6(1):238.

67. Xu C, Liu H, He Y, et al. Endothelial progenitor cells promote osteogenic differentiation in co-cultured with mesenchymal stem cells via the MAPKdependent pathway. Stem Cell Res Ther. 2020;11(1):537.

68. Caplan Al, Correa D. The MSC: an injury drugstore. Cell Stem Cell. 2011:9(1):11-5.

69. Rohban R, Pieber TR. Mesenchymal stem and progenitor cells in regeneration: tissue specificity and regenerative potential. Stem Cells Int. 2017;2017:5173732

\section{Publisher's Note}

Springer Nature remains neutral with regard to jurisdictional claims in published maps and institutional affiliations.

\footnotetext{
Ready to submit your research? Choose BMC and benefit from:

- fast, convenient online submission

- thorough peer review by experienced researchers in your field

- rapid publication on acceptance

- support for research data, including large and complex data types

- gold Open Access which fosters wider collaboration and increased citations

- maximum visibility for your research: over 100M website views per year
}

At BMC, research is always in progress.

Learn more biomedcentral.com/submissions 